\title{
NEW TOOLS FOR CULTURAL HERITAGE TOURISM: ACCESSIBLE VIRTUAL REALITY FOR MILAN'S BASILICA SANT'AMBROGIO
}

\author{
C. Pybus \\ Carleton Immersive Media Studio (CIMS), Carleton University, Canada - cpybus@ cims.carleton.ca
}

KEY WORDS: Cultural Heritage Dissemination, Game Engines, Virtual Reality, Interactivity, Accessibility

\begin{abstract}
:
Although virtual reality provides much new unexplored territory for presenting and disseminating cultural heritage, operating VR headsets and controllers remains challenging for new users. By taking advantage of the tools available within the Unreal Engine game engine, the ergonomics of the Oculus Rift motion controllers, and certain principles of storytelling; one can develop a set of strategies for making the use of virtual reality more accessible and intuitive, allowing for new possibilities in the dissemination of built cultural heritage to a general audience. Therefore his paper aims to provide concrete examples of how to produce more effective VR tools for cultural heritage dissemination.
\end{abstract}

\section{INTRODUCTION}

As part of ongoing efforts by the DABClab GIcarus research center of the Politecnico di Milano to study practices of informative modelling applied to the Basilica Sant'Ambrogio of Milan (Banfi et al, 2019), this paper describes the development of a prototypical virtual experience for cultural heritage tourism. Building off of digital assets created through pursuing a Scanto-BIM process (Brumana 2018) - survey data, a building information model (BIM), and historic documents were imported into Unreal Engine for the purpose of creating a prototypical project of public dissemination through virtual reality. Though this research focused on a specific investigation of VR using tethered headsets, it is only one part of a wider XR (extended reality) prototype which also considers networked applications of augmented and mixed reality on a variety of platforms. Even more specifically, this paper discusses the interactive methods developed during the research, a topic which currently lacks much precedent since VR technology has only been accessible since early 2016 .

In recent years the use of digital technologies for heritage recording has greatly expanded; however, questions remain as to how this burgeoning digital archive can be disseminated. The project discussed in this paper is a proposition for using digital heritage assets to communicate to the public the importance of a site, and it does so by making a prototype for an interactive virtual reality experience, facilitated by a game engine.

\subsection{The Basilica Sant'Ambrogio}

For the subject of this prototype, the Basilica Sant'Ambrogio in Milan was examined as a case study - a Romanesque basilica that dates back to the 4th century A.D and is one of Milan's most prominent monuments visited by thousands of tourists every year. Its many transformations over the centuries speak also about the urbanization of Milan and to a history of prominent Italian architects, changes which are legible within the visible accretion of construction techniques which characterize the building's morphology. That the basilica was built on an existing funerary area of unknown age only further emphasizes the remarkable length of time for which the site was and continues to be important (Stanga et al, 2017). This rich and complex history makes the basilica an ideal subject for examining how virtual technologies may help to 'reveal' history and inspire a curiosity, strengthening the information and content which can be provided to the visitor.

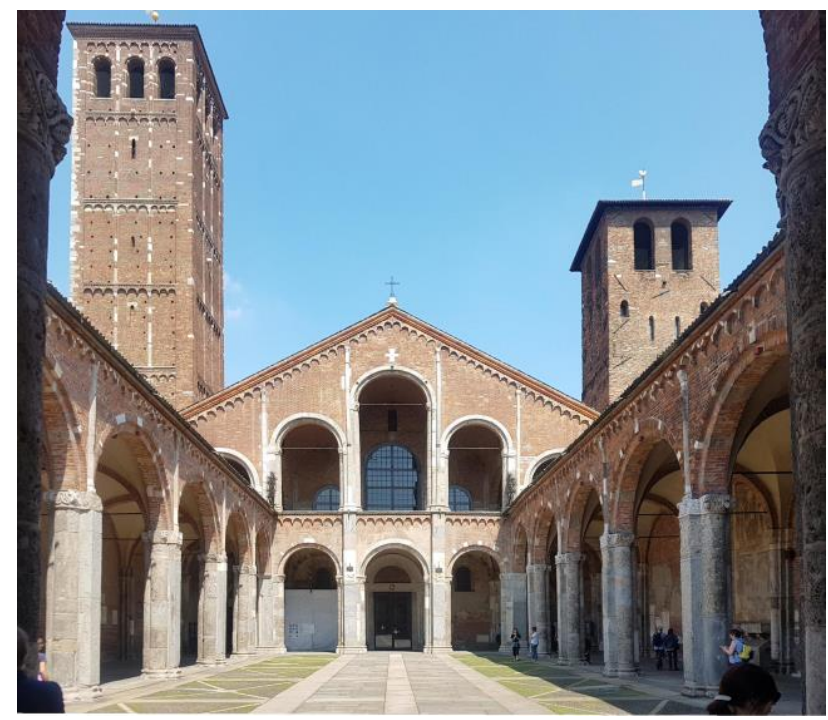

Figure 1. The Basilica Sant'Ambrogio.

\subsection{Game engines, the BIM to VR link}

BIM (Building Information Model) has many useful applications including design, conservation, and facilities management, and can be considered at its best as not only a 3D model, but as a spatialized database which may contain references to all required information of a built work. In the case of heritage BIM, the model has the potential to be the central location for referencing all historic documentation pertinent to a site. However, a fundamental challenge arises in the amount of investment required to operate BIM-both in terms of training and the purchase of proprietary software. Due to complex data structure, these challenges only multiply when attempting to export models from native BIM software to other applications. It is for these reasons that when investigating possibilities of how BIM might be made viewable in VR, the topic of game engines quickly arises as a means of enabling 
virtual reality, as it contains certain tools for facilitating diverse media formats, among other advantages..

Game engines have the potential to bridge an accessibility gap from sophisticated BIM software to a format that is more easily useable by a general user-basically they have a potential to provide a 'BIM link.' Originally created for the videogame industry to expedite the process of making sophisticated games, game engine provide a number of customizable tools which greatly assist efforts to visualize multimedia content and provide interactive functionality. Providing templates for creating VR experiences is only one example of the many tools that game engines provide. Moreover, game design favours intuitive easy-to-learn interactions, and the products of game engines are standalone applications rather than proprietary file types, both qualities which demonstrate the potential for greater accessibility of BIM content. Game engines can provide a practical means of viewing digital heritage assets in virtual reality, but even more generally they present many opportunities for rendering digital cultural heritage information more accessible and available for dissemination.

With an understanding of the relationship between BIM, game engines, and VR established, the specific questions which must be considered to create a VR experience come to the fore. There is a lack of convention for even basic considerations of how a user should navigate through virtual space. In addition to this lack of precedent, the unique qualities of VR, those that distinguish them from other mediums like text and film, are not yet well understood. One particular quality of VR is the notion of 'presence.' Wirth et al (2007) describe presence as a sense of 'being there' which arises when a person's perception is fooled into thinking they are somewhere else than their actual physical environment, and furthermore this quality has the ability to improve the effectiveness of educational simulations, and to intensify sensations of media effects. In this regard, it is thought that experiences in virtual reality have the potential to be more affective and memorable than other media.

\subsection{Digitally Assisted Storytelling}

Wirth et al remark that although virtual reality is particularly strong at stimulating presence, it can also occur in conventional media like books through mental construction in the imagination of the reader. Considering the above, this paper takes the position that sensations of presence can assist in fulfilling learning objectives, and that the case of the book example demonstrates how sense of presence can be further heightened through effective storytelling which stimulates the viewers imagination.

In a general sense, it is the position of the author that the thoughtful use of storytelling in experiences intended for cultural heritage dissemination will increase their effectiveness. For example, a common form of 'virtual tour' is a web-browser based application which allows the user to navigate through a series of 360 degree images which document a space, sometimes with 'hotspots' of special subjects. Unlike a 'reallife' tour, often these experiences are unguided and the user is prompted to explore content without any direction on where to begin and end their visit. The lack of guiding becomes a missed opportunity to engage the visitor and motivate them to browse the tour's contents, and it is a case which would benefit from storytelling.

In his essay The Storyteller (1969) Benjamin argues how storytelling has advantages over pure information and can be a potent method for preserving history. He writes that in storytelling, "...the psychological connection of the events is not forced on the reader. It is left up to him to interpret things the way he understands them, and thus the narrative achieves an amplitude that information lacks." Storytelling, therefore, actively engages the listener by arousing them subjectively and psychologically, making a story unforgettable. In this way, history - as story - is perpetuated not only within books but also as a collective memory. Complimenting VR's quality of presence, incorporating storytelling in a VR experience can make for a more compelling and memorable experience, and improving the dissemination of educational content in the case of cultural heritage tourism.

\subsection{Cultural Heritage VR Interactions}

In order to develop an understanding of effective interaction methods for VR, several existing recent projects will be examined in the next section. Section five goes into greater detail of five recommended strategies for VR cultural heritage projects. The strategies are intended to make the experience accessible to a general user who may have never used VR before and should therefore emphasize simple and intuitive interactions. They include an emphasis on gesture-based controls, recommendations for displaying graphic content, a strategy of presenting information similarly to familiar mediums, guiding through narrative, and considerations of hosting a VR experience on or away from a heritage site.

\section{STATE OF THE ART}

Precedent projects which use game engine and VR technology provide various insights to this project and inform its aims. This section will first outline methods for evaluating which game engine to choose, then consider several precedent projects for their technical and didactic merits.

\subsection{Game Engine Selection}

Independently from a conversation of built heritage, various authors argue for the importance of serious games for their ability to fulfil cognitive learning outcomes and motivate players (Michael and Chen 2005, Breuer et al. 2010). As distinguished from entertainment games, serious games are designed for a general audience and often require a simpler interface tailored to learning objectives (Petridis et al. 2010).

Notable among research of selecting game engines for serious games is the work of Petridis et al, which identifies several key criteria for comparison that are still useful despite changes in technology: audiovisual fidelity, functional fidelity, composability, accessibility, networking, and heterogeneity. 


\begin{tabular}{|c|c|}
\hline \multirow{2}{*}{ Audiovisual Fidelity } & Rendering \\
\cline { 2 - 2 } & Animation \\
\cline { 2 - 2 } Functional Fidelity & Sound \\
\cline { 2 - 2 } & Scripting \\
\cline { 2 - 2 } Composability & Supported Al Techniques \\
\cline { 2 - 2 } & Import / Export Content \\
\hline \multirow{3}{*}{ Accessibility } & Developer Toolkits \\
\cline { 2 - 2 } & Learning Curve \\
\cline { 2 - 2 } & Documentation and Support \\
\cline { 2 - 2 } & Licensing \\
\hline Networking & Cost \\
\hline Heterogeneity & Client Server / Peer-to-peer \\
\hline \multirow{2}{*}{ Online Community } & Multiplatform Support \\
\cline { 2 - 2 } & Fumber of Social Media Followers \\
\hline
\end{tabular}

Figure 2. Methodology for comparing game engines in serious games (Petridis et al 2010) with additional 'online community' criteria.

Subsequent research has attempted to further develop these categories in more specific cases, but can largely be considered inconsequential to the present discussion. A common theme emerges of stating that there are a plethora of game engines to choose from (Cowan and Kapralos 2014), but in practice there are two particularly outstanding options for developing VR experiences: Unreal Engine and Unity3D. Each were originally considered by Petridis et al in 2010 but have since become free to use, and the market has narrowed. Unity3D and Unreal engine have successfully overtaken much of their competition in the video game industry - as decisively stated by Autodesk in discontinuing its Stingray game engine (Autodesk Support 2008). Both engines have been used by the game industry since the early 2000's to produce hundreds of games and are very popular among game developers. Westhoven and Alexander (2015) state how their popularity is not to be underestimated considering that if one were to make a serious game project, software developers with a skill set including these two engines are widely available.

In addition to the accessibility of Unity3D and Unreal engines, the benefits of employing a widely used softwares present a lot of benefits, such as the support of an active online community (Patridis et al 2010, Wethoven and Alexander 2015). Considering the professional experience of CIMS with the development of game projects, this paper asserts that an active online community is essential, and therefore the category of 'online community' is elevated to Petridis' methodology as a seventh criteria - which can be quantitatively measured by examining numbers of social media followers and the rate of posts on community message boards.

For this project the most important requirements of the game engine were visual fidelity, simplicity of scripting, learning curve, and the availability of online resources including documentation and community support. These were a priority because of the importance of visual information and player interactivity, and because the project did not include a dedicated software developer on our team at the time.

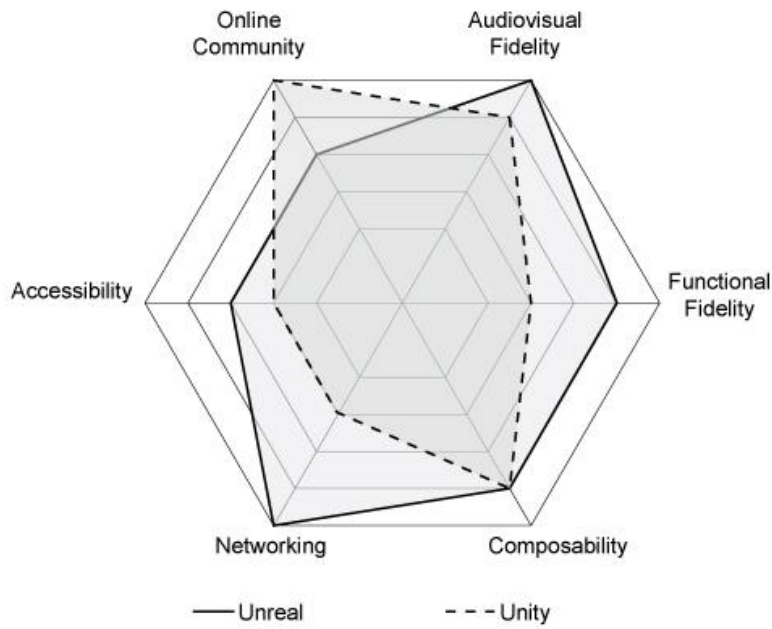

Figure 3. Graphic comparison of Unreal Engine and Unity 3D from the perspective of the project team.

Audiovisual fidelity favoured Unreal because it is simpler to achieve an impressive result with powerful hardware. The criteria of functional fidelity heavily favoured Unreal because the blueprints visual scripting system doesn't require a knowledge of writing code and is similar to the common architectural applications of Grasshopper and Dynamo. Composability was of equal interest for our uses, Unreal offers more streamlined tools for composing such as animation and sound editing, but these weren't important to development and was therefore not a decisive category. The criteria of accessibility in this case favoured Unreal only because of the team's aforementioned background knowledge of architectural software, and it provides more in-engine tools. The quality of online documentation for each is about equal, and they are both free to start but Unity restricts certain 'pro' features behind a paywall while allowing the developer to keep all revenues. Unreal is free to use and open source but claims revenues on commercial sales of developed applications. Networking was not a concern of this project. Regarding heterogeneity, the two softwares offer the same overall multiplatform support, but it's acknowledged that Unity is likely better for creating optimized mobile applications. While both have very active online communities, Unity's is currently larger and more invested in experimentation; however Unreal's is also large enough to provide examples of most common questions.

Overall the two engines are very competitive and anything done in one can likely be achieved in the other. They differ in what qualities are available 'out of the box,' in a general sense Unreal projects begin with a lot of complexity already enabled, whereas in Unity complexity must be added to a project. This likely reflects their origins in being originally designed for desktop computers and mobile devices respectively. Ultimately considering how competitive the two engines are, their selection depends more on the intentions of the project and the members of the team than on any notion of a 'better' game engine.

\subsection{Precedent Projects}

Although consumer VR headsets have only been available since spring 2016, there are already various examples of VR projects of built heritage. More broadly, there has been much useful research on the use of game engines for heritage conservation for non-VR applications. Considering the focus of this prototype is to create an experience for disseminating heritage 
research to interested tourists, one can distinguish informative precedents for either their technical or didactic merits.

In Serious Games for Relocation to a New Healthcare Facility by Merschbrock et al (2016), a completed BIM for a new healthcare facility was used to make a training program for employees to learn their tasks in preparation of moving to a new facility. This paper proves the case of using game engines for 'serious' application-demonstrating possibilities for the wide range of applications of game engine technology which leverages BIM assets.

The Rome Reborn project is a long-running digitization project housed at the University of Virginia in collaboration with the Politecnico di Milano. Started 1996, it sought to recreate an accurate 3D model of Rome from $320 \mathrm{AD}$ which was linked to an extensive dataset for archival purposes (Guidi et al. 2007). In August 2018 a selection of this project was released for purchase on the internet, making it publically accessible for the first time. This example is notable in that its $3 \mathrm{D}$ models are made manually rather than generated from recorded data. Similarly to BIM, these 3D models possess metadata which can also be imported to a game engine (Dylla et al. 2008).

Demonstrating an effective didactic approach, the project Bagan - Embracing the Future to Preserve the Past by Google Arts and Culture and CyArk (2008) illustrates the use of recorded heritage assets to create a guided interactive virtual tour of the ancient city of Bagan in Myanmar. This tour defines a general path for the user to follow, while providing them with more detailed optional content to explore freely as they proceed through the experience. The experience ends with a link to the full dataset of 3D scans provided by CyArk (2016), and is notable in how it presents different levels of detail for information. The experience is designed for a web browser which is viewable on desktop or mobile, and has optional VR components which operate through the WebVR specification.

Masterworks is another project completed by CyArk and released on the Oculus store in early 2018. Like the Bagan project it uses a curated selection of assets in order to create a didactic experience appealing to a general public (Harden 2018). Though it has more content and is more interactive than Bagan, it lacks the guided narrative of the Bagan project and is therefore less motivating to experience start to finish.

Two examples of effective educational VR experiences are Hold the World and Spheres. The former is an educational VR experience which exhibits photogrammetry of artifacts from London's Natural History Museum (Hayden 2018). It is fully interactive and narrated by a distinguished voice-actor, and further advances the use of storytelling in virtual experiences to promote didactic content. Spheres, though not about cultural heritage specifically, is currently one of the most effective examples of using narrative to inspire an interest of didactic content in VR, but in this case the subject is astronomy (Hayden 2018). Users visit different astronomical bodies across three experiences with visual and audio explanations. Professional writers and voice-actors were engaged with this project, and much greater creative interpretation was taken to exhibit its subject than other mentioned precedents, but this leads to a deeply memorable result.

\section{METHODS AND OBJECTIVES}

As stated the intention for this project was to share information collected by researchers to cultural heritage tourists while preserving the quality of the collected documentation. It is acknowledged that a tourist needs a certain degree of guidance to appreciate this unfamiliar information. Therefore, drawing from notable precedents, the communication of information within the virtual experience should be organized into a narrative structure to maintain visitor interest - a hierarchy where several specific heritage assets of the project can be used to tell a general story of Sant'Ambrogio, but additional information is available to the visitor if they are interested to view it. In this way the information is presented through a strategy of 'surface and depth.'

This strategy was used to organize how one would view the different spaces of Sant'Ambrogio. When a new visitor starts the experience they begin in a 'connecting space' which has limited content so as not to distract the visitor as they learn an overview of Sant'Ambrogio and VR. Then they proceed into richer spaces which show many more heritage assets. For this prototype we have only implemented a selection of the overall data of Sant'Ambrogio which emphasizes the history of the 'Barlonghe Vaults' within the central nave, but theoretically such an experience could be a way of viewing a complete database of digital heritage assets.

It should be emphasized that one of the benefits of using game engines is multiplatform support, and much of the application development can be abstracted from a specific platform for greater flexibility, allowing for an economy of efforts when porting to different platforms - for example desktop PC, AR, or mobile applications in addition to VR.

As stated one of the main advantages of game engines is they provide many tools for viewing and interacting with diverse media. However much time can be saved if the required media types are clearly determined before development because each type requires a different implementation strategy. In the case of the Sant'Ambrogio prototype, photogrammetric models, a Revit model, photographs, text, and audio were imported data types. It is also possible to work with 360 photos and video, however these media types were not implemented in this prototype. Though some of this data was collected through visits during the summer of 2018, the majority of documentation was already assembled through the research of Stanga and Spinelli (2017) and the previous research of the GIcarus lab (Brumana 1990, Banfi 2016).

\section{STRATEGIES FOR ACCESSIBILITY}

\subsection{Gesture-Based Controls}

The first strategy of accessibility is to base the controls for the VR experience off of familiar hand gestures and movements, rather than unfamiliar and awkward button presses. This intention was the main reason for choosing the Oculus Rift over the HTC Vive as the preferred VR headset, specifically because of the controller design. Both controllers draw much of their inspiration from the videogame industry, and they feature buttons, triggers, and control sticks organized in a way that would feel familiar to those that play video games. However there is a key difference in their ergonomic design - while the Vive controller 'wand-design' are intended to be held similarly to a television remote and used like a large laser-pointer, the Rift touch controllers have a 'resting hand' design that encourages the user to move their hands normally as if they weren't holding anything. Combined with sensors that detect the touch presence of fingers, the controllers are able to register familiar hand gestures like grasping an object, pointing one's 
index finger, making a fist, or giving a 'thumbs-up' sign. This ability to emulate gestures is absent from the current Vive design.

Rather than ask a user to 'press the $\mathrm{x}$ button,' which is further complicated by the fact that while wearing a VR headset a user can't see their hands, the experience can provide instructions like 'point at this object' which are commonly understood. Therefore, following the suggestion of Petridis et al to design for a non-gaming audience, interaction is not based on teaching the visitor how to use special controls, but simply on moving one's hands and gesturing intuitively.

In the realized experience, pointing becomes an important method of interacting with subjects in the virtual space. Pointing produces a 'laser-pointer' from the tip of the user's finger which, when pointed at a particular heritage asset, will present specific information about that asset to the user. In addition, certain objects can be 'picked-up' within the VR experience simply by reaching out to the object and grasping the controller as one would any object, allowing the virtual object to be handled for close visual inspection, and can be passed from hand to hand.

Finally, rather than ask the user to press buttons on the controller, when requiring input from the user buttons appear as virtual objects near them within the game space. 'Touching' the button in virtual space by moving one's hand to the correct location is all that is required to interact with them. In effect, none of the buttons on the controller are used at any point.

For all this to work smoothly it is important to have quality hand models used within the virtual experience so the user can confirm their gesture with visual feedback. The default hand models from the Unreal VR template were decidedly lacking, in that they only demonstrated opening and closing and lacked animations for more specific gestures. However the 'Avatar SDK' provided by Oculus was integrated into the project, producing a more realistic representation of the user's hand movements.

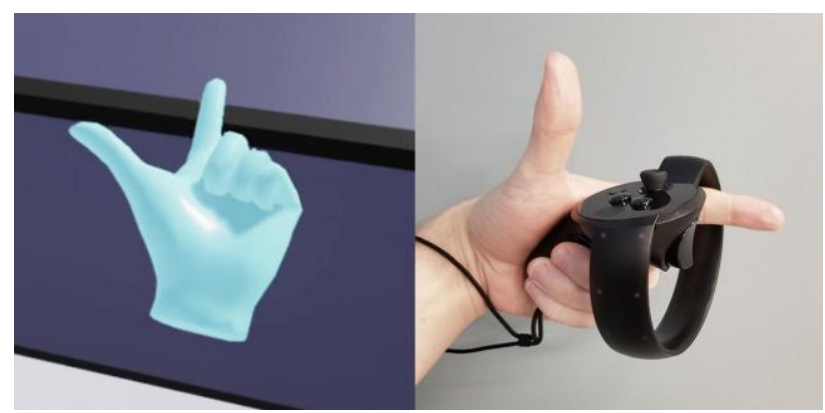

Figure 4. Interaction using realistic hand gestures.

\subsection{Immersive Graphic Content}

It was essential that this project communicate familiar types of graphic content (images, text) to the visitor to fulfill learning objectives, but VR doesn't provide recognizable 2D presentation surfaces like paper and computer monitors. All VR content must digitally manifest in $3 \mathrm{D}$ space, so the conventions of presenting 2D content must be reconsidered. Three immersive strategies of presenting graphic information were developed: 'in space' - graphics content appears as if floating in 3D space; 'in HUD' - graphics content appears overlaid over the user's vision as if they were wearing augmented reality glasses; and 'hand-attached' - where information is presented in a 'virtual notebook' like using a tablet computer or holding a clipboard.

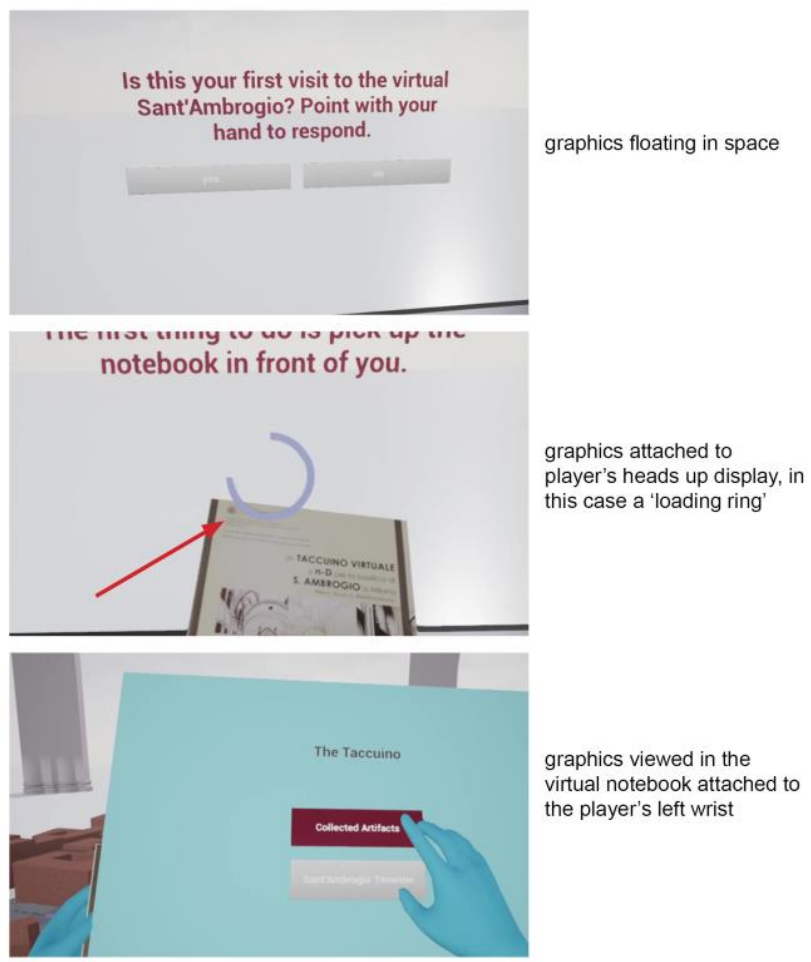

Figure 5. Three immersive strategies for presenting graphical content in VR: in space, in HUD, and wrist-attached.

The choice of which strategy to use in a given situation was dependent on narrative intention and the level of information needed, with the virtual notebook best suited to presenting conventional 2D content with minimal adaptation to VR conditions.

The HUD (heads-up display) approach of attaching information directly to the user's vision initially seemed the most promising, but upon testing it was found to be visually confusing and should be used sparingly. It feels quite discomforting to have text and graphics attached to one's vision regardless of location and orientation. since the eyes are accustomed to focusing on subjects in the center of the cone of vision. Therefore the HUD should likely remain empty, with occasional graphic information placed temporarily in the center of vision only as needed.

\subsection{New But Familiar Interfaces}

The concept of a virtual notebook for Sant'Ambrogio which contains references to all relevant information about the building was first described by Stanga and Spinelli as a possible computer tablet application (2017). The concept was adapted to VR following a previous example developed by the author originally for 2D screens (Pybus 2018). Notably although the concept has been adapted to VR, it actually still functions like a tablet application, with the intention of maintaining familiar controls to the user.

The idea of using the virtual notebook as a tablet, but in VR, is informed by Ulmer's notions of new technological development (1994). The interactivity of the virtual notebook does not seek 
to be completely new, but based off analogies to existing technology that are likely to be familiar to the user-in this case the swiping and tapping of a tablet computer. In this regard any interaction designed for the VR experience should be 'like another action' that the user is already familiar with.

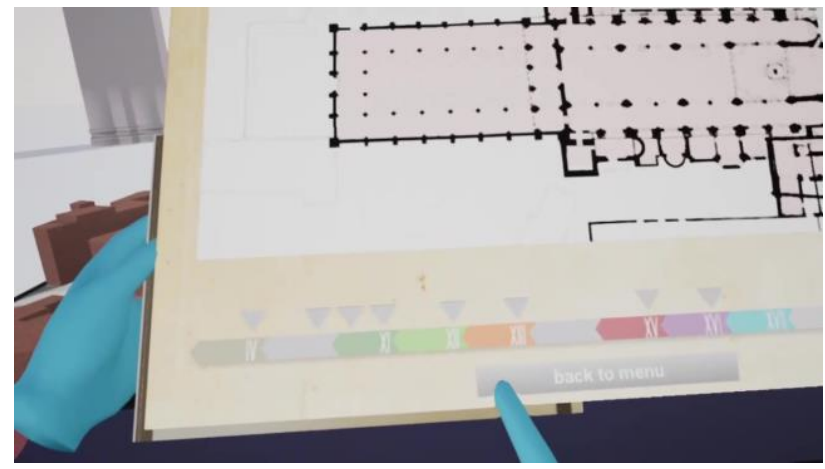

Figure 6. The virtual notebook provides detailed information about the heritage assets in the virtual experience and is used in a similar way to a physical tablet computer.

\subsection{Guiding Through Narrative}

The previously mentioned Bagan project by Google and CyArk is already a very clear example of this strategy of guiding through narrative. Although virtual experience can provide access to many kinds of content at any time, and indeed having lots of diverse content is likely favourable for cultural heritage dissemination, too much content without a clear direction of what one should do can produce an aimless experience that the user is unmotivated to spend time with. This pertains once again to the author's critique of the aforementioned approach to online virtual tours, it is recommended there should be at least a minimum amount of guiding narrative to give the experience a sense of beginning and end.

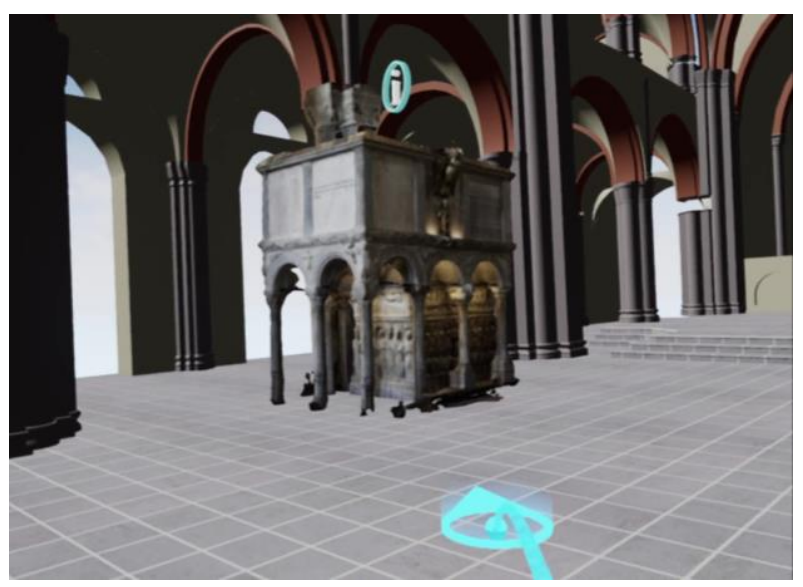

Figure 7. The visitor is directed to interact with a specific model to progress to the next space, but other heritage assets are optionally viewable along the way.

The Bagan project presented the viewer with an overall path and map to guide them between the different temples that were documented, but at any time the viewer can stop to explore the hotspots available at any particular point along the narrative path. In that way the narrative and the content support each other, providing either direction or distraction at the whims of the curiosity of the visitor. In the case of Sant'Ambrogio, particular subjects within a scene are given specific emphasis one at a time to facilitate a linear experience and a sense of progression. Meanwhile, additional heritage content is available in the space and viewable should the visitor choose to explore it.

\subsection{On-Site and Off-Site Implementation}

Although not realized in this project it is important to consider where the visitor will be physically interacting with the VR experience. Although there are already a number of cultural heritage projects available for purchase on VR app stores, suggesting an experience that the visitor enjoys by themselves at home, there are notable examples of VR tours being installed on cultural heritage sites and used to complement a physical guided tour. Although a VR headset blocks out the view of the user's physical location, there is still a temporal quality of viewing a VR experience either just before or just after visiting the represented space in real life.

In the VR Kiosk project completed by CIMS in summer 2017, five VR headsets were installed across the street from Canada's Parliament in Ottawa, Canada which showed historic recreations of spaces a tourist was about to his visit (Graham et al, 2017). In this way a tourist could experience a space in its current state while with the VR headset they were able to visualize the spaces as they were in the past. Another project demonstrating this concept is 'Beyond the Castle,' a VR experience currently exhibited in Sforza Castle, Milan. This experience recreates several historic events that took place at Sforza during the renaissance and shows the visitor what the castle would have looked like at this time. The visitor is taken on a tour of these actual spaces before beginning the experience.

In the case of Sant'Ambrogio a hybrid approach is proposed, where the VR experience can be temporarily installed on site as part of a tour of the building. However the application should ideally then be adapted to be able to stand by itself, such that it could be distributed on the internet, allowing for broader public dissemination to those who might like to experience it at home if they lack the opportunity to travel to Milan. It should be noted that exhibiting a VR project on-site requires employing a staff to assist visitors in using the VR headsets, a fact that has been previously demonstrated in the exhibiting of both the VR Kiosk and Spheres projects.

\section{CONCLUSION}

The consideration of the use and adaptation of digital cultural heritage assets as a digital experience mediated through game engines can be efficient in an virtual reality effort for cultural heritage dissemination. Assets which were prepared for archival purposes can be given a second life through educational experiences as described in this paper. Through game engines, one can create a 'BIM link' to virtual reality. The use of virtual reality and its associated quality of presence can further enhance a project's ability to fulfill learning outcomes, especially if combined with storytelling to engage the visitor, as demonstrated by various examples. Unreal Engine was chosen as the game engine for this project mainly because of its graphical fidelity and simplicity of scripting, but Unity3D could have also produced a comparable result. The development of this prototypical VR experience for Sant'Ambrogio highlighted five strategies to make VR accessible to a general audience, and to raise the quality of VR cultural heritage projects, including an emphasis on gesture-based controls, recommendations for displaying graphic content, a strategy of presenting information similarly to familiar mediums, guiding through narrative, and 
considerations of hosting a VR experience on or away from a heritage site.

Although this paper focused on a specific VR application with the Oculus Rift, the assets which were prepared are also ready to be used in a desktop, or AR application, demonstrating an economy of efforts that may be possible with the multiplatform potential of game engine software.

Finally, in consideration of adapting this prototype to a complete experience, it would be recommended to bring additional disciplines into the process. Such a virtual experience would benefit from having artistic direction to guide the scenario design, and computer developers to expedite the development process. That said, for a small project Unreal Engine is remarkable in its ability to provide a robust toolkit to an informed but non-expert user.

\section{ACKNOWLEDGEMENTS}

1. This research was conducted with the support of the Social Sciences and Humanities Research Council of Canada as part of the New Paradigms New Tools internship program of the Carleton Immersive Media Studio.

2. The author wants to thank DABClab GIcarus research center (Department of Architecture, Built Environment and Construction Engineering), Politecnico di Milano (Italy) hosting the internship (R. Brumana, F. Banfi, C. Stanga).

3. Data from surveying, modeling, HBIM generation, interpretation and communication of the Basilica di Sant'Ambrogio in Milan have been provided and supported by the on-course GAMHer project: Geomatics Data Acquisition and Management for Landscape and Built Heritage in a European Perspective, PRIN, Progetti di Ricerca di Rilevante Interesse Nazionale - Bando 2015, Prot. 2015HJLS7E. (Sc. Res. POLIMI Unit R. Brumana), with the contribution of Fabrizio Banfi (GEO) and Chiara Stanga (RES).

4. Thanks to Adam Weigert and Miquel Reina Ortiz for their help creating with documentation and photogrammetry of the site.

\section{REFERENCES}

Autodesk Support, "Changes to Autodesk Stingray FAQ," AutodeskKnowledge Network, 24-Jan-2018. [Online]. Available: https://knowledge.autodesk.com/searchresult/caas/sfdcarticles/sfdcarticles/stingray-end-of-salefaq.html. [Accessed: 05-Mar-2019].

F. Banfi, M. Previtali, C. Stanga, and R. Brumana, "a LayeredWeb Interface Based on Hbim and $360^{\circ}$ Panoramas for Historical, Material and Geometric Analysis," International Archives of the Photogrammetry, Remote Sensing and Spatial Information Sciences, vol. 42, no. 2/W9, 2019.

F. Banfi, "Building Information Modelling - A Novel Parametric Modeling Approach Based on 3D Surveys of Historic Architecture," in Digital Heritage. Progress in Cultural Heritage: Documentation, Preservation, and Protection, 2016, pp. 116-127.

W. Benjamin, "The Storyteller," in Illuminations: Essays and Reflections, New York: Schocken, 1969.
J. S. Breuer and G. Bente, "Why so serious? On the relation of serious games and learning," Eludamos. Journal for Computer Game Culture, vol. 4, no. 1, pp. 7-24, Apr. 2010.

R. Brumana, 'Sant'ambrogio's Basilica in Milan: a study on photogrammetric surveys in the S. Vittore in Ciel D'oro's dome," in Close-Range Photogrammetry Meets Machine Vision, 1990, vol. 1395, p. 139539.

R. Brumana, P. Condoleo, A. Grimoldi, F. Banfi, A. G. Landi, and M. Previtali, "HR LOD based HBIM to detect influences on geometry and shape by stereotomic construction techniques of brick vaults," Appl Geomat, vol. 10, no. 4, pp. 529-543, Dec. 2018.

CIMS, "Heritage Passages: Bytown and the Rideau Canal," Virtual Museums of Canada, 19-Mar-2013. [Online]. Available: http://www.virtualmuseum.ca/virtual-exhibits/exhibit/heritagepassages-bytown-and-the-rideau-canal/. [Accessed: 05-Mar2019].

B. Cowan and B. Kapralos, "A Survey of Frameworks and Game Engines for Serious Game Development," in 2014 IEEE 14th International Conference on Advanced Learning Technologies, 2014, pp. 662-664.

CyArk, "Bagan Open Heritage Request," CyArk, 08-Jun-2016. [Online]. Available: https://www.cyark.org/projects/bagan/overview. [Accessed: 03Mar-2019].

K. Dylla, B. Frischer, P. Müller, A. Ulmer, and S. Haegler, "Rome reborn 2.0: A case study of virtual city reconstruction using procedural modeling techniques," Computer Graphics World, vol. 16, no. 6, pp. 62-66, 2008.

Google Arts and Culture, "Bagan - Embracing the Future to Preserve the Past," Experiments With Google, May-2018. [Online]. Available: https://experiments.withgoogle.com/bagan. [Accessed: 03-Mar-2019].

K. Graham et al., "The VR kiosk," in Digital Cultural Heritage, Springer, 2018, pp. 324-336.

G. Guidi, B. Frischer, and I. Lucenti, "Rome Reborn Virtualizing the ancient imperial Rome," in Workshop on 3D Virtual Reconstruction and Visualization of Complex Architectures, 2007.

S. Hayden, “VR Tour App 'MasterWorks' Uses Photogrammetry to Bring You to 4 Fully Explorable Heritage Sites," Road to VR, 17-Feb-2018. .

S. Hayden, "David Attenborough's VR Experience 'Hold the World' Lands on Rift," Road to VR, 03-Nov-2018. .

S. Hayden, “Award-Winning VR Space Experience 'SPHERES' Lands on Rift," Road to VR, 14-Nov-2018. .

C. Merschbrock, A. K. Lassen, T. Tollnes, and B. E. Munkvold, "Serious games as a virtual training ground for relocation to a new healthcare facility," Facilities, vol. 34, no. 13/14, pp. 788808, Oct. 2016.

D. R. Michael and S. L. Chen, Serious Games: Games That Educate, Train, and Inform. Muska \& Lipman/Premier-Trade, 2005. 
Oculus / Developers, "Guidelines for VR Performance Optimization," Oculus Documentation. [Online]. Available: https://developer.oculus.com/documentation/pcsdk/latest/conce pts/dg-performance-guidelines/. [Accessed: 11-Jan-2019].

P. Petridis, I. Dunwell, S. de Freitas, and D. Panzoli, "An Engine Selection Methodology for High Fidelity Serious Games," in 2010 Second International Conference on Games and Virtual Worlds for Serious Applications, 2010, pp. 27-34.

C. Pybus, "Intangible Territories: The Island of Salty Dreams," Text, Carleton University, 2018.

C. Stanga, C. Spinelli, R. Brumana, D. Oreni, R. Valente, and F. Banfi, "A nd virtual notebook about the basilica of S. Ambrogio in Milan: information modeling for the communication of historical phases subtraction process," The International Archives of Photogrammetry, Remote Sensing and Spatial Information Sciences, vol. 42, p. 653, 2017.

C. Stanga and C. Spinelli, "Un taccuino virtuale a nD per la basilica di Sant'Ambrogio a Milano. Rilievo, ricerca, rielaborazione," 2015.

G. L. Ulmer, Heuretics: The Logic of Invention. JHU Press, 1994.

M. Westhoven and T. Alexander, "Towards a Structured Selection of Game Engines for Virtual Environments," in Virtual, Augmented and Mixed Reality, 2015, pp. 142-152.

W. Wirth et al., "A Process Model of the Formation of Spatial Presence Experiences," Media Psychology, vol. 9, no. 3, pp. 493-525, May 2007. 